\title{
Éditorial
}

\section{Les questions d'extraire ou de ne pas extraire apparaissent déjà, dans les ouvrages de Pierre Fauchard', sous la forme de quelques recommandations empiriques :}

\footnotetext{
$\ll D$ e ne jamais extraire des dents de lait trop tôt, car il se rencontre quelquefois des dents de lait qui ne tombent
} De ne jamais extraire les dernières molaires de lait pour pallier un manque d'espace, afin de ne pas causer un autre dérangement plus considérable qu'il n'aurait peut-être été auparavant » (p. 911).

Extraire nécessairement toutes dents surnuméraires... extraire toute dent permanente qui se trouve hors de rang, qui blesse la langue ou les joues, qui choque la vue par sa difformité et qui ne peut être logée dans le rang des autres dents. Cette règle est générale et ne souffre aucune exception si la place pour la canine n'est pas assurée il faut l'extraire (p. 163).

Si une dent est cassée il faut ôter sa racine avant que de rapprocher les dents voisines par le moyen d'un fil.

Si les dents penchées ne peuvent être remises en place, faute d'un espace suffisamment large pour les loger on est obligé d'ôter une des dents qui sont penchées, pour distribuer sa place à toutes celles qui en ont besoin («le chirurgien-dentiste », II. 103) etc.

Où en sont les praticiens, après deux siècles d'observations et d'études scientifiques?

Bien que les décisions de traitement soient prises au cas par cas, nous pensons qu'ils présentent encore une attitude plutôt subjective face à ces questions d'extractions.

1. Fauchard P., le chirurgien-dentiste ou traité des dents, Paris, Édition Julien Prelat, réédition 1961. 
Plusieurs enquêtes, auprès d'orthodontistes pourtant chevronnés, illustrent comment leur aversion ou leur préférence à extraire l'emporte sur la rigueur de jugements formels.

On a comparé le taux d'extraction estimé « au jugé » par des praticiens avec celui obtenu par l'analyse arithmétique de leurs fichiers. Ils ne concordaient pas, puisque le premier taux se situait entre 5 à $87 \%$, alors que le second était compris entre 25 et $80 \%$ !

Dans une autre enquête, on réinterrogea, à un mois d'intervalle, dix orthodontistes, sur leur décision d'extraire. Malgré ce court laps de temps, les résultats étaient déjà différents ! Une étude a montré que, dans la décision d'extractions, les mesures téléradiographiques ou sur moulages avaient moins de poids que «le sentiment initial », etc.

Chacun sait qu'au cours du temps, beaucoup d'orthodontistes sont influencés par un environnement d'approbations tacites initiées par des professionnels charismatiques.

La décision d'extraire n'échappe pas aux incertitudes et aux revirements qui expriment la difficulté qu'ont les praticiens à établir une stratégie de traitement sur des bases scientifiques incontestables.

La recherche légitime de la stabilité ou de l'excellence des résultats, tant fonctionnels qu'esthétiques, dépend souvent du choix d'extraire ou pas.

Malheureusement, l'orthodontiste ne dispose pas de moyens de prédictibilité suffisamment fiables tant la variabilité individuelle est élevée dans les domaines qui sont les siens comme la croissance, la coopération...

Après chaque traitement, des praticiens peuvent regretter d'avoir extrait ou non, faute de ne pas avoir suffisamment bien maîtrisé l'ensemble des paramètres prévisionnels nécessaires. Par conséquent, et pour chaque nouveau patient, il ne peut faire l'économie d'une réactivation de son raisonnement dans un espace d'incertitudes et d'efforts sans cesse renouvelés :

«Les dieux avaient condamné Sisyphe à rouler sans cesse un rocher jusqu'au sommet d'une montagne d'où la pierre retombait par son propre poids. Ils avaient pensé avec quelque raison qu'il n'est pas de punition plus terrible que le travail inutile et sans espoir»

"Le mythe de Sisyphe », A. Camus

Michel Amoric

edpdentaire
La Librairie Garancière, l'excellence dentaire

Retrouvez notre sélection experte d'ouvrages en médecine bucco-dentaire

\section{Librairie Garancière}

